\title{
Equally-Weighted Quadrature Formulas for Inversion Integrals
}

In a previous article [1] the author considered Gaussian-type quadrature formulas for the numerical evaluation of inversion integrals $\frac{1}{2 \pi i} \int_{c-i \infty}^{c+i \infty} \frac{e^{p}}{p} F(p) d p$, where an $n$-point formula was exact whenever $F(p)$ was a polynomial of degree $(2 n-1)$ in $1 / p$. In this present note we consider equally weighted (i.e., Chebyshev type) quadrature formulas of the form

$$
\frac{1}{2 \pi i} \int_{c-i \infty}^{c+i \infty} \frac{e^{p}}{p} F(p) d p=\frac{1}{n} \sum_{j=1}^{n} F\left(p_{j}\right)
$$

where (1) is exact whenever $F(p)$ is any polynomial of degree $n$ in $1 / p$. The analogous set of equally weighted quadrature formulas for evaluating infinite integrals that are direct Laplace transforms has already been considered in one of the author's earlier papers [2]. Similar to the derivation given there, it is easily seen here that the well-known relation

$$
\frac{1}{2 \pi i} \int_{c-i \infty}^{c+i \infty} \frac{e^{p}}{p}\left(\frac{1}{p}\right)^{r} d p=\frac{1}{r !}
$$

by choosing $r=0$, establishes the factor $1 / n$ outside the summation in (1), and the choice of $r=1,2, \cdots, n$ establishes the following necessary and sufficient conditions on $p_{j}$, in order that (1) should hold whenever $F(p)$ is an arbitrary $n$th degree polynomial in $1 / p$

$$
\sum_{j=1}^{n}\left(\frac{1}{p_{j}}\right)^{r}=\frac{n}{r !}, \quad r=1,2, \cdots, n
$$

Following the usual methods [2], one determines for the $n$-point case of (1) the coefficients of the polynomials $\phi_{n}(z)$ whose zeros $z_{j}$ are the reciprocals of the required points $p_{j}$. Thus from $\phi_{n}(z)=z^{n}+a_{1} z^{n-1}+a_{2} z^{n-2}+\cdots+a_{n-1} z+a_{n}$, the coefficients $a_{k}$ are found successively from

(3) $k a_{k}+a_{k-1} \sum_{j=1}^{n} z_{j}+a_{k-2} \sum_{j=1}^{n} z_{j}{ }^{2}+\cdots+a_{1} \sum_{j=1}^{n} z_{j}^{k-1}+\sum_{j=1}^{n} z_{j}{ }^{k}=0$,

$$
k=1,2, \cdots, n .
$$

Below are the general formulas for the first ten coefficients $a_{1}, a_{2}, \cdots, a_{10}$ for any $n$, having meaning, of course, only for an $a_{k}$ where $k \leq n$ 
198 EQUALLY-WEIGHTED QUADRATURE FORMULAS FOR INVERSION INTEGRALS

$$
\begin{aligned}
& a_{1}=-n \text {, } \\
& a_{2}=\frac{n^{2}}{2}-\frac{n}{4}, \\
& a_{3}=-\frac{n^{3}}{6}+\frac{n^{2}}{4}-\frac{n}{18} \text {, } \\
& a_{4}=\frac{n^{4}}{24}-\frac{n^{8}}{8}+\frac{25}{288} n^{2}-\frac{n}{96} \text {, } \\
& a_{5}=-\frac{n^{5}}{120}+\frac{n^{4}}{24}-\frac{17}{288} n^{3}+\frac{7}{288} n^{2}-\frac{n}{600}, \\
& a_{6}=\frac{n^{6}}{720}-\frac{n^{5}}{96}+\frac{43}{1728} n^{4}-\frac{25}{1152} n^{3}+\frac{1507}{259200} n^{2}-\frac{n}{4320} \text {, } \\
& a_{7}=-\frac{n^{7}}{5040}+\frac{n^{6}}{480}-\frac{13}{1728} n^{5}+\frac{13}{1152} n^{4}-\frac{1741}{259200} n^{3}+\frac{53}{43200} n^{2}-\frac{n}{35280}, \\
& a_{8}=\frac{n^{8}}{40320}-\frac{n^{7}}{2880}+\frac{61}{34560} n^{6}-\frac{7}{1728} n^{5}+\frac{17627}{4147200} n^{4}-\frac{3779}{2073600} n^{3} \\
& +\frac{15787}{67737600} n^{2}-\frac{n}{322560} \text {. } \\
& a_{9}=-\frac{n^{9}}{362880}+\frac{n^{8}}{20160}-\frac{7}{20736} n^{7}+\frac{19}{17280} n^{6}-\frac{22289}{12441600} n^{5} \\
& +\frac{2887}{2073600} n^{4}-\frac{487471}{1097349120} n^{3}+\frac{12317}{304819200} n^{2}-\frac{n}{3265920}, \\
& a_{10}=\frac{n^{10}}{3628800}-\frac{n^{9}}{161280}+\frac{79}{1451520} n^{8}-\frac{11}{46080} n^{7}+\frac{69353}{124416000} n^{6} \\
& -\frac{11347}{16588800} n^{5}+\frac{2237339}{5486745600} n^{4}-\frac{26791}{270950400} n^{3} \\
& +\frac{590383}{91445760000} n^{2}-\frac{n}{36288000} .
\end{aligned}
$$

The first ten polynomials $\phi_{n}(z)$ are

$$
\begin{aligned}
& \phi_{1}(z)=z-1, \\
& \phi_{2}(z)=z^{2}-2 z+\frac{3}{2}, \\
& \phi_{3}(z)=z^{3}-3 z^{2}+\frac{15}{4} z-\frac{29}{12}
\end{aligned}
$$




$$
\begin{aligned}
& \phi_{4}(z)=z^{4}-4 z^{3}+7 z^{2}-\frac{62}{9} z+\frac{289}{72} \\
& \phi_{5}(z)=z^{5}-5 z^{4}+\frac{45}{4} z^{3}-\frac{535}{36} z^{2}+\frac{1805}{144} z-\frac{1627}{240}, \\
& \phi_{6}(z)=z^{6}-6 z^{5}+\frac{33}{2} z^{4}-\frac{82}{3} z^{3}+\frac{481}{16} z^{2}-\frac{4537}{200} z+\frac{27769}{2400}, \\
& \phi_{7}(z)=z^{7}-7 z^{6}+\frac{91}{4} z^{5}-\frac{1631}{36} z^{4}+\frac{4417}{72} z^{3}-\frac{106351}{1800} z^{2} \\
& +\frac{5302619}{129600} z-\frac{18044381}{907200} \\
& \phi_{8}(z)=z^{8}-8 z^{7}+30 z^{6}-\frac{628}{9} z^{5}+\frac{4037}{36} z^{4}-\frac{3277}{25} z^{3}+\frac{922919}{8100} z^{2} \\
& -\frac{2922187}{39690} z+\frac{145511171}{4233600} \\
& \phi_{9}(z)=z^{9}-9 z^{8}+\frac{153}{4} z^{7}-\frac{407}{4} z^{6}+\frac{3027}{16} z^{5}-\frac{103911}{400} z^{4}+\frac{650239}{2400} z^{3} \\
& -\frac{8499571}{39200} z^{2}+\frac{41478457}{313600} z-\frac{1514611753}{25401600} \\
& \phi_{10}(z)=z^{10}-10 z^{9}+\frac{95}{2} z^{8}-\frac{1280}{9} z^{7}+\frac{43235}{144} z^{6}-\frac{170381}{360} z^{5}+\frac{1490251}{2592} z^{4} \\
& -\frac{17363761}{31752} z^{3}+\frac{415158089}{1016064} z^{2}-\frac{3255225203}{13716864} z+\frac{142324922009}{1371686400} .
\end{aligned}
$$

The values of $p_{j}$ and the zeros $z_{j} \equiv 1 / p_{j}$ of the above polynomials $\phi_{n}(z)$, for $n=1(1) 10$, are given in Table 1 .

The zeros $z_{j} \equiv 1 / p_{j}$ of $\phi_{n}(z)$ were calculated for $n=3,4, \cdots, 10$, by first obtaining an initial approximation, using a procedure that had been employed upon the Univac Scientific Computer (ERA 1103) at the Convair Digital Computing Laboratory. The initial approximations to the complex zeros were then used to construct approximate real quadratic factors, which were refined by Bairstow's method (Milne [3], Olver [4]), using only a desk calculator. The initial approximations to the real zeros were refined by Newton's method. All factors of $\phi_{n}(z)$ were checked by four different formulas (see [4], p. 414). Also a final functional check was performed upon the values of $1 / p_{j}$ by substituting into equation (2) for $n=1$ (1)10, and $r=1(1) n$.

The 8-decimal values of $p_{j}$ and $1 / p_{j}$ in Table 1 are guaranteed as far as the seventh decimal place. But they are believed to be correct to within around two units in the eighth decimal place for $n=1(1) 7$ and have a high probability of being correct to within several units in the eighth decimal place for $n=8(1) 10$. 
TABLE 1. $p_{j}$ and $1 / p_{j}$

\begin{tabular}{|c|c|c|c|c|c|c|c|c|c|}
\hline$n$ & $j$ & \multicolumn{4}{|c|}{$p_{j}$} & \multicolumn{4}{|c|}{$1 / p_{j}$} \\
\hline 1 & 1 & 1.00000 & $000+$ & .00000 & $000 i$ & 1.00000 & $000+$ & .00000 & $000 i$ \\
\hline 2 & 1,2 & .66666 & $667 \pm$ & .47140 & $452 i$ & 1.00000 & $000 \mp$ & .70710 & $678 i$ \\
\hline 3 & $\begin{array}{c}1,2 \\
3\end{array}$ & $\begin{array}{l}.46343 \\
.62485\end{array}$ & $\begin{array}{l}318 \pm \\
778+\end{array}$ & $\begin{array}{l}.66891 \\
.00000\end{array}$ & $\begin{array}{l}55 i \\
00 i\end{array}$ & $\begin{array}{r}.6 \\
1.6\end{array}$ & $\begin{array}{l}792 \mp \\
417+\end{array}$ & $\begin{array}{l}11 \\
00\end{array}$ & $000 i$ \\
\hline 4 & $\begin{array}{l}1,2 \\
3,4\end{array}$ & $\begin{array}{l}.31209 \\
.54603\end{array}$ & $\begin{array}{l}699 \pm \\
449 \pm\end{array}$ & $\begin{array}{l}.78 \\
.22\end{array}$ & $\begin{array}{l}870 i \\
497 i\end{array}$ & $\begin{array}{r}.4 \\
1.5\end{array}$ & $\begin{array}{l}772 \mp \\
228 \mp\end{array}$ & .6 & $\begin{array}{l}277 i \\
456 i\end{array}$ \\
\hline 5 & $\begin{array}{c}1,2 \\
3,4 \\
5\end{array}$ & $\begin{array}{l}.19029 \\
.46724 \\
.53392\end{array}$ & $\begin{array}{l}304 \pm \\
697 \pm \\
634+\end{array}$ & $\begin{array}{l}.86260 \\
.36843 \\
.00000\end{array}$ & $\begin{array}{l}499 i \\
448 i \\
000 i\end{array}$ & $\begin{array}{r}.24387 \\
1.31966 \\
1.87291\end{array}$ & $\begin{array}{l}201 \mp \\
923 \mp \\
752+\end{array}$ & $\begin{array}{r}1.0 \\
.0\end{array}$ & $\begin{array}{l}814 i \\
000 i\end{array}$ \\
\hline 6 & $\begin{array}{l}1,2 \\
3,4 \\
5,6\end{array}$ & $\begin{array}{l}.08786 \\
.39416 \\
.49826\end{array}$ & $\begin{array}{l}626 \pm \\
727 \pm \\
825 \pm\end{array}$ & $\begin{array}{l}.92009 \\
.46819 \\
.14769\end{array}$ & $\begin{array}{l}404 i \\
799 i \\
920 i\end{array}$ & $\begin{array}{l}85 \\
29 \\
84\end{array}$ & $\begin{array}{l}254 \mp \\
916 \mp \\
830 \mp\end{array}$ & & 4 \\
\hline 7 & $\begin{array}{c}1,2 \\
3,4 \\
5,6 \\
7\end{array}$ & $\begin{array}{r}-.00076 \\
.32727 \\
.45588 \\
.49224\end{array}$ & $\begin{array}{l}496 \pm \\
973 \pm \\
935 \pm \\
949+\end{array}$ & $\begin{array}{l}.96470 \\
.54346 \\
.25464 \\
.00000\end{array}$ & $\begin{array}{l}825 i \\
944 i \\
118 i \\
000 i\end{array}$ & $\begin{array}{r}-.00082 \\
.81317 \\
1.67190 \\
2.03149\end{array}$ & $\begin{array}{l}196 \mp \\
581 \mp \\
107 \mp \\
016+\end{array}$ & $\begin{array}{r}1.35033 \\
.93385 \\
.00000\end{array}$ & $\begin{array}{l}217 i \\
175 i \\
569 i \\
000 i\end{array}$ \\
\hline 8 & $\begin{array}{l}1,2 \\
3,4 \\
5,6 \\
7,8\end{array}$ & $\begin{array}{r}-.07902 \\
.26601 \\
.41223 \\
.47182\end{array}$ & $\begin{array}{l}919 \pm \\
917 \pm \\
251 \pm \\
912 \pm\end{array}$ & $\begin{array}{r}1.00066 \\
.60293 \\
.33698 \\
.10911\end{array}$ & $\begin{array}{l}480 i \\
762 i \\
985 i \\
533 i\end{array}$ & $\begin{array}{r}-.07843 \\
.61252 \\
1.45409 \\
2.01181\end{array}$ & $\begin{array}{l}500 \mp \\
402 \mp \\
421 \mp \\
677 \mp\end{array}$ & $\begin{array}{r}1.38829 \\
1.18868 \\
.46525\end{array}$ & $\begin{array}{l}762 i \\
595 i \\
329 i\end{array}$ \\
\hline 9 & $\begin{array}{c}1,2 \\
3,4 \\
5,6 \\
7,8 \\
9\end{array}$ & $\begin{array}{r}-.14919 \\
.20966 \\
.36931 \\
.44525 \\
.46815\end{array}$ & $\begin{array}{l}526 \pm \\
304 \pm \\
455 \pm \\
659 \pm \\
071+\end{array}$ & $\begin{array}{r}1.03046 \\
.65149 \\
.40305 \\
.19444 \\
.00000\end{array}$ & $\begin{array}{l}752 i \\
353 i \\
392 i \\
915 i \\
000 i\end{array}$ & $\begin{array}{r}-.13761 \\
.44761 \\
1.23580 \\
1.88616 \\
2.13606\end{array}$ & $\begin{array}{l}844 \mp \\
307 \mp \\
348 \mp \\
975 \mp \\
428+\end{array}$ & $\begin{array}{r}.95050 \\
1.39088 \\
1.34870 \\
.82371 \\
.00000\end{array}$ & $\begin{array}{l}835 \\
424 \\
244 \\
403 \\
000\end{array}$ \\
\hline 10 & $\begin{array}{l}1,2 \\
3,4 \\
5,6 \\
7,8 \\
9,10\end{array}$ & $\begin{array}{r}-.21284 \\
.15754 \\
.32790 \\
.41610 \\
.45488\end{array}$ & $\begin{array}{l}773 \pm \\
418 \pm \\
360 \pm \\
417 \pm \\
509 \pm\end{array}$ & $\begin{array}{r}1.05570 \\
.69213 \\
.45764 \\
.26374 \\
.08636\end{array}$ & $\begin{array}{l}953 i \\
469 i \\
025 i \\
950 i \\
297 i\end{array}$ & $\begin{array}{r}-.18351 \\
.31266 \\
1.03454 \\
1.71443 \\
2.12187\end{array}$ & $\begin{array}{l}682 \mp \\
794 \mp \\
187 \mp \\
371 \mp \\
330 \mp\end{array}$ & $\begin{array}{r}.91023 \\
1.37363 \\
1.44386 \\
1.08670 \\
.40285\end{array}$ & $036 i$ \\
\hline
\end{tabular}

The refinement of the factors and zeros of $\phi_{n}(z)$ by Bairstow's method was done by Mrs. Genevieve Mullin Kimbro.

Herbert E. SAlzer

CONVAIR Astronautics

San Diego, California

1. H. E. SALZER, "Orthogonal polynomials arising in the numerical evaluation of inverse Laplace transforms," MTAC, v. 9, 1955, p. 164-177.

2. H. E. SAlzER, "Equally weighted quadrature formulas over semi-infinite and infinite intervals," Jn. Math. and Physics, v. 34, 1955, p. 54-63.

3. W. E. Milne, Numerical Calculus, Princeton University Press, Princeton, New Jersey, 1949 , p. 53-57.

4. F. W. J. OLVER, "The evaluation of zeros of high-degree polynomials," Roy. Soc. London, Phil. Trans., v. 244, Ser. A, 1952, p. 385-415. 